\section{The aftermath of Core-}

\section{Collapse Supernovae}

Roger A. Chevalier, Department of Astronomy, University of Virginia, USA

$T$ he lifecycles of stars considerably more massive than the sun show that they end in spectacular events in which the core collapses and the outer parts are ejected in a tremendous explosion. The Rosetta stone for the end states of such stars has been the Crab Nebula and its association with the supernova of 1054 (SN 1054) (Fig. 1). The amorphous radiation from the interior of the nebula was identified as synchrotron radiation from relativistic particles in the 1950's, but the source of the particles was not known until the discovery of a central pulsar, a rapidly rotating magnetized neutron star, in 1968. The spin-down of the central pulsar, with its $33 \mathrm{msec}$ period, was shown to account for the energetic requirements of the nebula. The synchrotron radiation implies the presence of a relativistic bubble of magnetic fields and particles, which are responsible for sweeping the surrounding stellar ejecta into a shell.

In a young (age $1000 \mathrm{yr}$ ) supernova remnant, the pulsar is expected to affect the inner part of the exploded star, while the outer part of the star interacts with the surrounding medium. In the Crab Nebula, we see the inner pulsar bubble, but we have not detected the interaction with the surrounding medium. This has been a long-standing puzzle with the Crab.

For many years, the Crab pulsar and the older Vela pulsar were the only pulsars that could be clearly linked to the explosive remnants of supernovae. That situation has recently changed; the number of pulsars with surrounding remnants is greater than a dozen. A combination of factors has allowed this advance, but particularly important has been the launch of X-ray space observatories with excellent spatial resolution and sensitivity (ASCA, Chandra, XMM-Newton). These observations have shown that a young pulsar in a supernova remnant is typically surrounded by a pulsar-generated nebula of relativistic particles and magnetic fields generated by the central pulsar as in the Crab Nebula. In many cases, the interaction with the surrounding medium is also observed.

At the same time as these discoveries, there have been advances in our understanding of the final evolution of massive stars and the various types of core collapse supernovae. Core collapse is believed to occur in stars with an initial mass $>8 \mathrm{M}_{\odot}$, where $\mathrm{M}_{\odot}$ is the mass of the sun. Above that mass, the final outcome depends on the rate of mass loss and the core development at the center of the star. Observations of stellar mass loss and evolutionary studies of massive stars show that mass loss rates increase with increasing initial stellar mass (and luminosity). These differences in presupernova mass loss can be linked to the different supernova types and, ultimately, to the different kinds of core collapse remnants. The aim of this paper is to describe these links and the implications for the end states of massive stars. Full details and references can be found in Chevalier (2005).

\section{Supernova types}

Stars just above the limit for core collapse are expected to end their lives as cool, extended red stars, with most of their hydrogen envelope intact. The explosion of these stars leads to a luminous supernova over an extended time (100 days) during which the light is from the heated hydrogen envelope. This type of explosion can be identified with Type IIP supernovae, where the II implies that hydrogen is present in the spectrum and the $\mathrm{P}$ stands for a plateau in the light curve. Above some initial mass, around $25 \mathrm{M} \odot$, most of the hydrogen envelope is lost during the stellar evolution, but there is enough left to give an extended envelope at the time of explosion. These objects can be identified with Type IIL (linear light curve) in which there is a more rapid drop from light maximum than in the IIP case. Above a higher mass, around

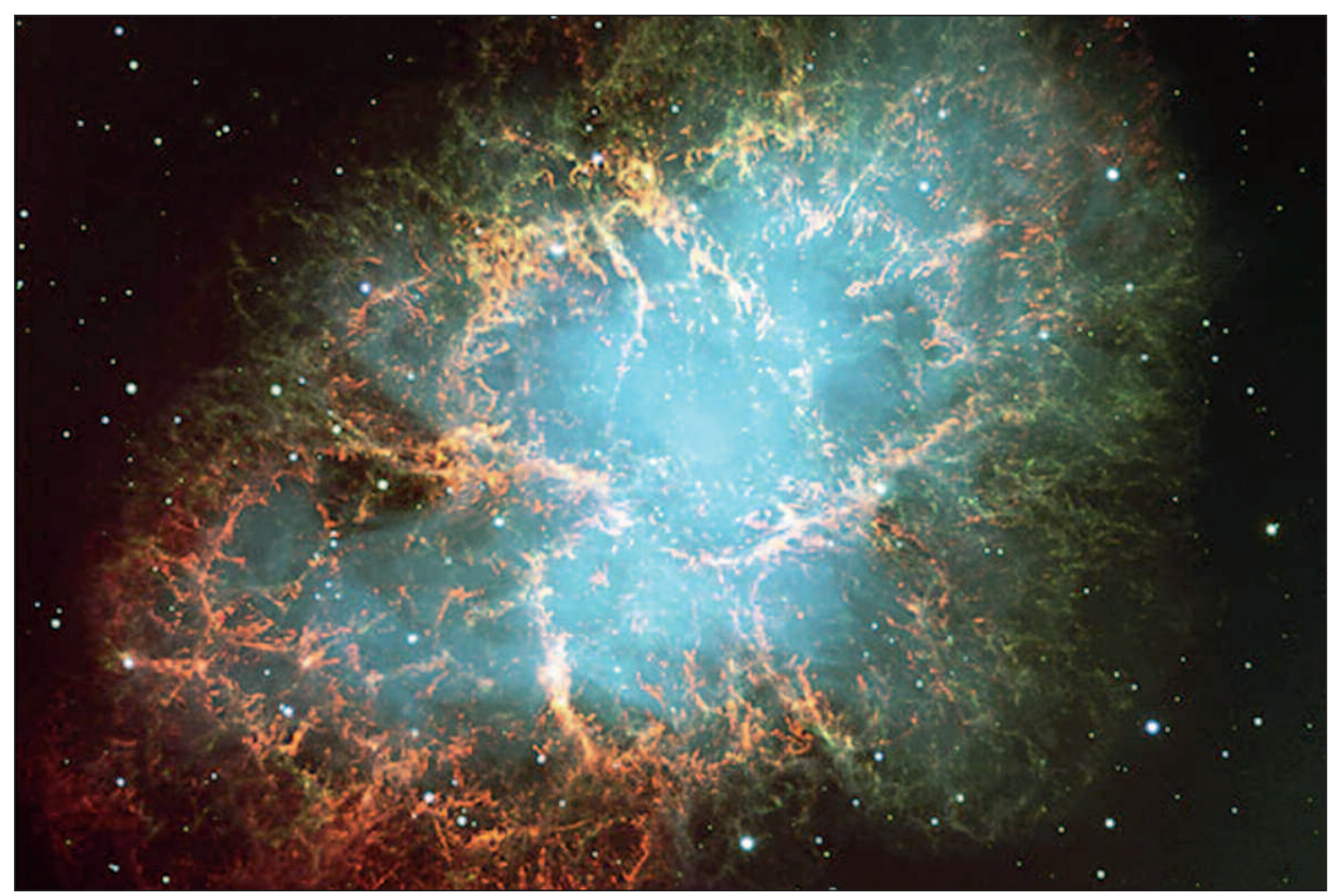

4 Fig. 1: Optical image of the Crab Nebula. The reddish, filamentary emission is gas from the exploded star that has been swept out by the pulsar bubble. The bluish, smooth emission is synchrotron radiation from relativistic particles that have been accelerated by the pulsar power (European Southern Observatory, Very Large Telescope). 


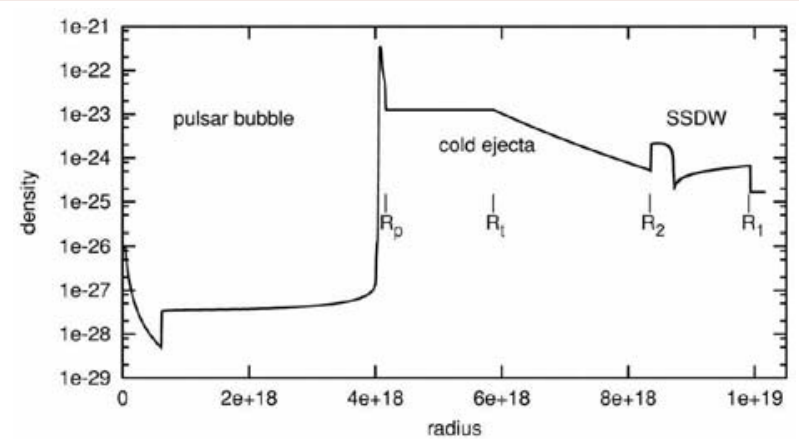

$\Delta$ Fig. 2: A one-dimensional model for a young supernova remnant (age $500 \mathrm{yr}$ ); the density is in $\mathrm{gm} \mathrm{cm}^{-3}$ and the radius in $\mathrm{cm}$. Starting from radius $=0$, there is a freely expanding pulsar wind, a region of shocked pulsar wind (pulsar bubble), a swept up shell of ejecta bounded by a shock front at Rp, a region of cold, freely expanding ejecta, and a region of hot gas (SSDW) bounded by a reverse shock at $\mathrm{R}_{2}$ and $a$ forward shock at $\mathrm{R}_{1}$ (from Blondin, Chevalier \& Frierson 2001).

$35 \mathrm{M}_{\odot}$, the hydrogen envelope is completely lost during the stellar evolution. These stars are observed as relatively compact, hot stars. The explosions of these stars can be identified with Type Ib or Ic supernovae, which do not have hydrogen in their spectra.

The rate of star formation is dominated by the lower mass stars, so that the expected rate of Type IIP supernovae is about 10 times the rate of Type IIL and 5 times the rate of Type Ib/c. Observational data are not yet sufficiently detailed to determine the relative rates. However, the above results are for single massive stars. A significant fraction of massive stars are in binary systems and mass transfer and loss due to binary effects could influence the relative rates of various core collapse supernovae. The importance and detailed effects of binary evolution remain one of the most uncertain aspects of stellar evolution.

\section{Supernova properties}

The central core collapse in a massive star triggers an explosion of the rest of star, by a mechanism that remains poorly understood. A mechanism involving transfer of energy from neutrinos has been favored, but has not yet provided a convincing model for an explosion; this has recently led to the exploration of other possible mechanisms, involving rapid rotation and magnetic fields. In the immediate aftermath of the explosion, the behavior of the layers near the collapsed core depend on the explosion mechanism, but, as one moves out in the star, the shock acceleration of the stellar layers is primarily determined by the explosion energy. The eruption of the explosion shock from stellar surface gives a burst of hard, typically ionizing, radiation. This emission has not yet been directly observed from a supernova, although the effects of such emission can be seen in the nearby supernova SN 1987A.

The time for the shock front to traverse the star is $<1$ day, and is followed by a period in which the internal energy of the explosion is converted to kinetic energy. After about a week, the expansion tends towards a homologous, free expansion. The density profile that is set up by the expansion is fairly flat in the central region and has a steep power law profile in the outer parts where the shock front has accelerated through the decreasing density layers of the star. The density of gas at a given velocity drops as $\mathrm{t}^{-3}$ due to the 3-dimensional expansion. The typical energy of a core collapse supernova is $10^{51} \mathrm{ergs}\left(10^{22} \mathrm{H}\right.$ bombs $)$.
As described above, the supernova types IIP, IIL, and Ib/c have increasing amounts of mass loss, which has implications for the composition distribution of the freely expanding ejecta. In a Type IIP supernova, most of the explosion energy ends up in the $\mathrm{H}$ envelope, whereas in the other types, the energy is in heavy element rich material. If the explosion were strictly radial and spherically symmetric, the explosion would have a set of composition layers, like an onion, with the heaviest elements at the center. Actually, composition discontinuities can be accompanied by strong density gradients; when the denser, interior material is decelerated by the outer lower density material in the explosion, the interface is subject to Rayleigh-Taylor instabilities. The result is that clumps of heavy elements go out to high velocities, while bubbles of light elements move to low velocities. The effects of this redistribution of elements can be seen in the late spectra of supernovae, when the ejecta have become optically thin.

The deceleration of an inner layer is accompanied by a reverse shock wave, a shock front that eventually moves back toward the center of the explosion. Material brought back to the center in this way can be important for fallback onto the central compact object. Some fallback is expected for gas that is marginally bound to the compact object. In high mass stars, including most single stars that are expected to end as Type IIL or Ib/c supernovae, the fallback is expected to be strong, leading to the formation of black holes. In other cases, the accreted mass is $<0.1 \mathrm{M} \odot$. The nature of the fallback can depend on the type of supernova. Even if the neutron star is born with slow rotation, the inward mixing of material can bring higher angular momentum material into the vicinity of the compact object.

Finally, different types of circumstellar medium, built up by stellar mass loss, are expected in the different types of supernovae. The speed of a stellar wind is related to the escape velocity from the star, so that extended red supergiant stars, which are the expected supernova progenitors of stars with hydrogen envelopes, have relatively slow winds $\left(\mathrm{v}_{\mathrm{w}}=10-20 \mathrm{~km} \mathrm{~s}^{-1}\right)$. These stars thus have a relatively high surrounding wind density, Úw, because in a steady spherical flow $\rho_{\mathrm{w}}=(\mathrm{dM} / \mathrm{dt}) /\left(4 \pi \mathrm{r}^{2} \mathrm{v}_{\mathrm{w}}\right)$, where $\mathrm{dM} / \mathrm{dt}$ is the mass loss rate and $r$ is the radius from the center. The value of $\mathrm{dM} / \mathrm{dt}$ depends primarily on the luminosity of the star, which is higher for the higher initial mass stars. Thus, for single stars, the Type IIL supernovae are expected to have the densest circumstellar winds, while the Type IIP are at lower density. Binary interaction can also lead to dense winds in Type IIL supernovae. The expansion of the wind is ultimately limited by the pressure of the surrounding medium. The high ram pressure in the denser winds means that they can expand out 5 parsecs $(1$ parsec $=3.26$ light-years $=3.09 \times 10^{18} \mathrm{~cm}$ ) or more from the progenitor star. Type Ib/c supernovae are thought to have more compact progenitors with higher velocity winds, $\mathrm{v}_{\mathrm{w}}=1000 \mathrm{~km} \mathrm{~s}^{-1}$. Their mass loss rates are comparable to those expected around Type IIL supernovae, but the high wind velocity leads to a relatively small surrounding density.

These expectations have been borne out by observations of extragalactic supernovae at ages up to a few years. The interaction with the surrounding wind can be observed at radio and X-ray wavelengths, as well as optical in the case of a dense wind. The interaction with the wind gives rise to hot shocked gas and relativistic particles that are probably produced by the shock wave acceleration mechanism. The relativistic electrons radiate synchrotron emission in the magnetic field of the shocked region. The early absorption of radio synchrotron emission and the synchrotron luminosity provide indicators of the circumstellar density. The thermal emission from the hot gas at X-ray wavelengths 
provides another estimate of the circumstellar density. The new X-ray observatories (Chandra, XMM-Newton) have made the detection of nearby extragalactic supernovae routine. The circumstellar densities inferred around the various types of supernovae are roughly in accord with expectations.

\section{Pulsar wind nebulae}

The presence of a pulsar inside of a supernova generates a bubble of relativistic fluid inside of the supernova. The spinning neutron star loses rotational energy through a magnetized wind that moves out from the pulsar at highly relativistic velocity. In order to be compatible with the slow moving gas outside of the bubble, the wind must pass through a shock wave that decelerates the wind and converts the wind power to internal energy (Fig. 2). This type of model was first applied to the Crab Nebula, where it was found that the position of the wind termination shock was compatible with the observed position of filaments in the synchrotron emission. A description of the pulsar wind nebula as a bubble of relativistic fluid, with adiabatic index $4 / 3$, provides an initial approximation to the pulsar nebula.

The pulsar wind nebula expands into the freely expanding ejecta of the supernova (cold ejecta in Fig. 2). If the pulsar power input is steady and the inner density profile of the supernova can be described by a power-law in radius, $\rho \sim \mathrm{r}^{-\mathrm{m}}$ (with $\mathrm{m}$ about 1 ), the interaction is self-similar and the radius of the wind bubble increases as a power law in time, $R \sim \mathrm{t}^{(6-\mathrm{m}) /(5-\mathrm{m})}$. The radius accelerates because the bubble is sweeping up gas that is expanding away from it. The fact that a light fluid is accelerating a denser fluid gives rise to the Rayleigh-Taylor instability, which is the likely origin of the filamentary structure observed in the Crab Nebula (Fig. 1). The acceleration of filaments in the Crab is directly indicated by the fact that the expansion age of the filaments is less than the age of the parent supernova SN 1054.

If the pulsar maintains its power and the bubble is able to expand into the steeply dropping part of the supernova density profile, the relativistic fluid can break out and fill the volume out to the shell of circumstellar interaction. The energy from the pulsar required to do this is comparable to the kinetic energy of the supernova, $\sim 10^{51}$ ergs, which places an upper limit on the initial rotational period of the pulsar, about 9 msec. No observed nebulae appear to have undergone this rapid expansion, implying that they do not begin building a relativistic bubble at such a high spin rate. If pulsar nebulae do not reach the breakout phase, they are either in an early phase of steady power input (assuming no evolution of the pulsar magnetic field), or the pulsars have spun down from their initial rotation rates. The evolutionary status of a nebula can be estimated by comparing the kinetic and internal energies of the nebula to $(\mathrm{dE} / \mathrm{dt}) \mathrm{t}$, where $\mathrm{dE} / \mathrm{dt}$ is the pulsar power output and $t$ is the age of the nebula.

\section{Young supernova remnants with pulsars}

The presence of a pulsar wind nebula inside of a supernova remnant gives valuable diagnostic information on the nature of the supernova. In the case of the Crab Nebula, the emission from the shell of ejecta shows the presence of hydrogen moving at about

Fig. 3: X-ray image of G292.0+1.8. The pulsar nebula with its central pulsar (135 msec period) is the blue region to the left of the center. The brighter emission is from gas heated by the interaction with the circumstellar medium. The radius of the remnant is about 7 parsecs. (NASA/Chandra X-ray (Observatory/Smithsonian Astrophysical Observatory)
$2000 \mathrm{~km} \mathrm{~s}^{-1}$, as expected in a Type IIP supernova. The outer edge of the Crab Nebula is observed to be in essentially free expansion, as expected for expansion into the supernova ejecta. The observed radius and acceleration of the gas shell are consistent with acceleration by the pulsar wind bubble. No circumstellar interaction around the Crab Nebula has been observed, despite extensive multiwavelength efforts. In a Type IIP model, this can be attributed to expansion into the low density wind bubble left from the main sequence phase of evolution of the massive star progenitor. This is the initial, and longest, phase of evolution for a massive star; the star has a strong, fast wind that can create a low density region around the star. Early interaction with the red supergiant wind would have led to hot gas that adiabatically expands and becomes unobservable. Although this scenario is plausible, a definitive case has not yet been made and deeper searches for emission surrounding the Crab Nebula are needed.

The appearance of G292.0+1.8 is different in that it shows strong circumstellar interaction, as well as a pulsar nebula (Fig. 3). The radius of the emitting region, $7 \mathrm{pc}$, and the emitting mass, several $\mathrm{M}_{\odot}$, are consistent with a dense surrounding wind, suggesting a Type IIL explosion. Optical observations of supernova ejecta moving at about $2000 \mathrm{~km} \mathrm{~s}^{-1}$ show that it is hydrogen-poor and heavy element rich, as expected for this kind of supernova. The estimated age is 3200 years.

There is enough information on 9 young nebulae that contain pulsars to estimate their ages, initial pulsar periods, and supernova types. The initial periods of the pulsars are inferred to be in the range $10-100 \mathrm{msec}$, but the periods are not correlated with the supernova type. The same is true for the pulsar magnetic fields deduced from the assumption of spin-down by magnetic dipole radiation. This would be surprising in a single star model for the stellar evolution because the core properties depend on the mass of the progenitor. However, if close binary evolution plays a role in the mass loss determining the supernova type, the lack of correlation is not surprising.

\section{SN 1987A and conclusions}

All the objects discussed to this point have ages of about $10^{3}$ years. The best opportunity we have to study the aftermath of a younger explosion is the nearby supernova SN 1987A in the LMC. Neutrinos were detected from the supernova in 1987, showing that a compact object formed in the explosion. However,

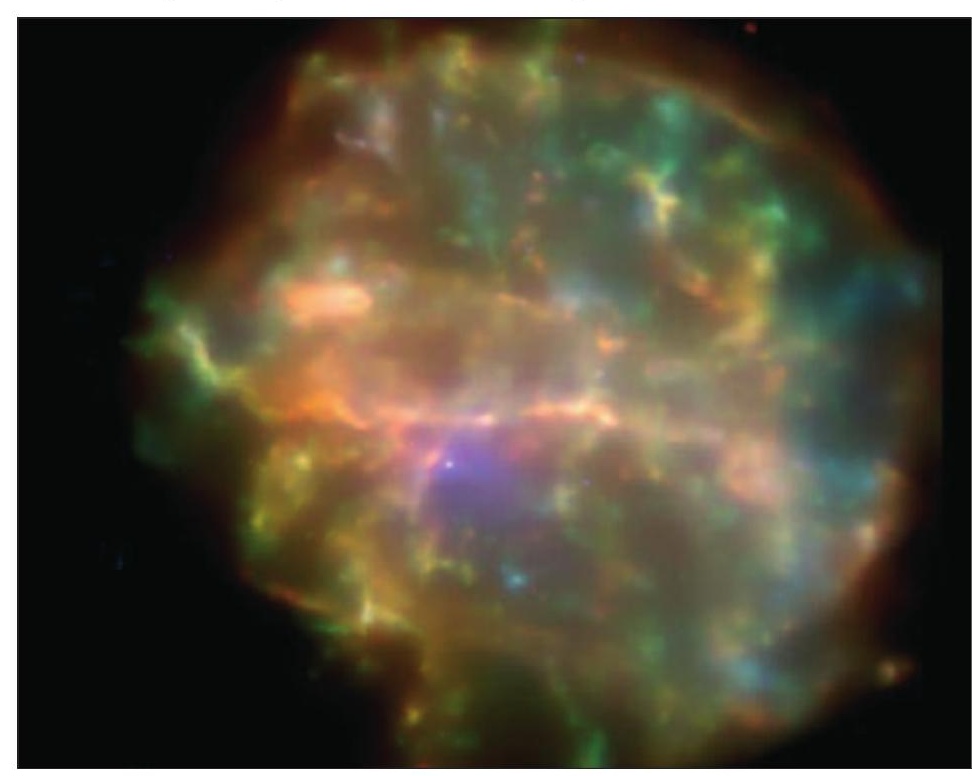




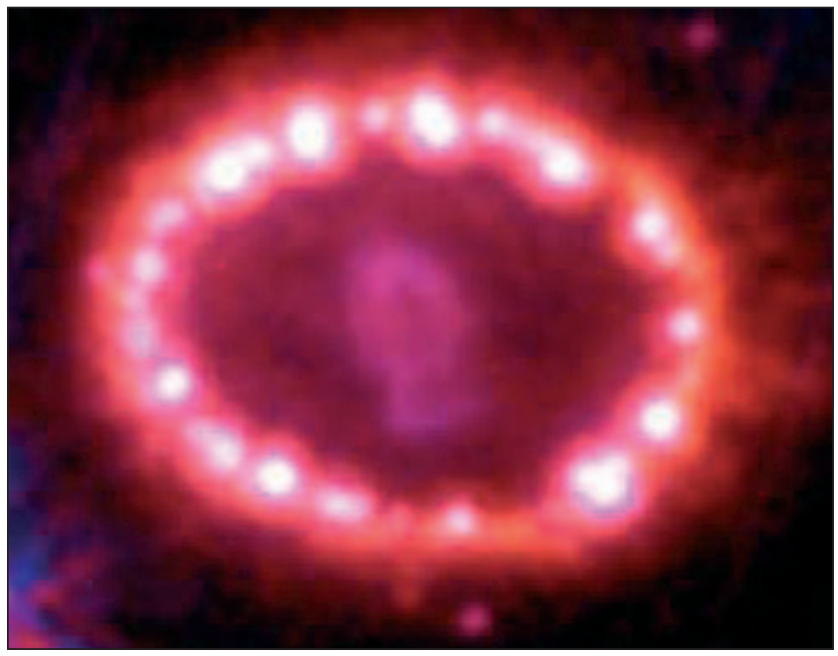

$\Delta$ Fig. 4: Optical image of SN 1987A in the Large Magellanic Cloud, a companion galaxy to our Milky Way Galaxy (taken 28 November, 2003). The ring, believed to be a circular ring with a radius of 0.2 pc observed at an angle, was created by presupernova mass loss. The bright spots on the ring are where the supernova shock front is driving shock waves into the dense ring material. The asymmetric central region is supernova debris heated by radioactive decays. (NASA/Hubble/STScl)

multiwavelength observations since that time have failed to give any evidence for the compact object. The optical emission from the region of the explosion can be attributed to power input by radioactive decays of ${ }^{44} \mathrm{Ti}$ (Fig. 4). Current limits on the luminosity of any compact object are orders of magnitude smaller that the power output of the Crab pulsar, and are lower than expectations for accretion onto a quiet neutron star. Accretion onto a black hole, with low radiative efficiency, is one possibility, but a quiet neutron star with little mass around it cannot be ruled out. Fortunately, the emitting supernova ejecta are becoming more extended and more optically thin with time, so it is just a matter of waiting until the mystery at the center reveals itself.

The increasing power of space and ground-based observatories is giving us the possibility of following through the supernova explosion to the effects of a central compact object and circumstellar interaction around massive stars. These phenomena tell us about fundamental astrophysical issues: the ejection of heavy elements and energy into the interstellar medium and the early evolution of neutron stars. The number of well-observed objects is still small; as it increases, we will have a clearer view of the diverse paths of massive star death.

\section{About the author}

Roger Chevalier is W.H. Vanderbilt Professor of Astronomy at the University of Virginia. He is a member of the National Academy of Sciences and was awarded the 1996 Dannie Heineman Prize for Astrophysics for his research on supernovae.

\section{References}

Blondin, J.M., Chevalier, R.A., Frierson, D.M., 2001, "Pulsar Wind Nebulae in Evolved Supernova Remnants,” Astrophys. J., 563, 806

Chevalier, R. A. 2005, "The Young Remnants of Core Collapse Supernova Remnants and Their Supernovae," Astrophys. J., 619, 839

\section{Lithuanian Physical}

\section{Society}

\author{
Zenonas Rudzikas, Rasa Kivilšienè
}

$T$ he history of physics teaching and physics research in Lithuania goes back to the sixteenth century, when in 1579 the University was founded in Vilnius, the oldest university in Eastern Europe. Physics was always taught there together with the other natural sciences. The newest achievements in physics were presented to the students with some delay, mainly caused by low speed of travel of the newest scientific literature to this then remote part of Europe.

In 1773 the Rector of Vilnius University, M.Poczobut-Odlanicki, presented a draft for an Academy of Sciences in Vilnius; however, perhaps due to the complicated political situation in that region at that time, the initiative did not become a reality.

In the $19^{\text {th }}$ century physics was taught in the old Vilnius University in a particularly modern way, because at that time there existed especially good contacts of local professors with their colleagues in France, England and other European countries. It also became common to buy modern equipment from these and other countries. The Vilnius University observatory also had strong links with a number of observatories in Western Europe.

The recent roots of the Lithuanian Physical Society (LPS) originated in the Lithuanian Scientific Society (LSS) founded in 1907, when Lithuania was still the so-called North-West Region of the Russian tsarist empire. The core of the LSS consisted of educated Lithuanians of various specialities, physicists and engineers included, who had the desire to collect and preserve the Lithuanian ethnographical cultural artefacts, to contribute to the education of the people, and to build up the national self-esteem. Much attention was paid to collecting data on geology, botany, zoology and other branches of science, to writing and publishing textbooks and popular scientific literature.

The LSS had its own library, its collection of manuscripts, an ethnographic archive, and museum. It also edited the journal "Lietuvių tauta" ("Lithuanian Nation") (1907-1936).

Special attention was paid to writing and publishing textbooks. During 1915-1920 about 100 textbooks for Lithuanian schools were written and published. When the Vilnius region was occupied by Poland in 1920, the LSS became very active in opening the new university in Kaunas, then the temporal capital of Lithuania.

In 1928 the Society of the Students of Physics and Mathematics was founded at Kaunas University, and in 1931 the Lithuanian Society of Natural Scientists. The latter was active up to 1940. It had about 200 members representing the physical, mathematical, chemical, biological, geological and medical sciences.

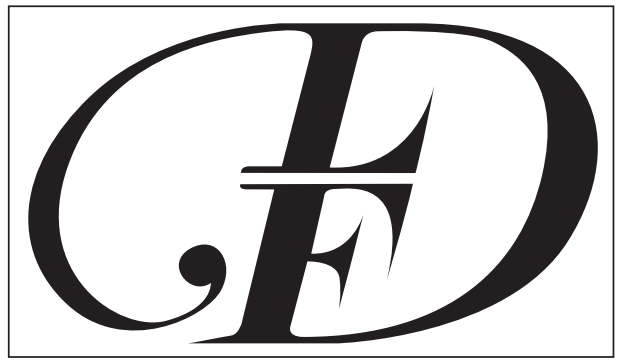

4 Fig. 1: Emblem of LPS 